\title{
Begabte Schwindlerinnen. Über die Inszenierungen der Lüge bei Ljudmila Ulickaja
}

Charmantes affabulatrices. La mise en scène du mensonge chez Ludmila

Oulitskaïa

Gifted Cheats. On the Staging of Lies in Lyudmila Ulickaja's Women's Lies

\section{Christiane Solte-Gresser}

\section{(2) OpenEdition}

\section{Journals}

Édition électronique

URL : http://journals.openedition.org/ceg/1554

DOI : $10.4000 /$ ceg. 1554

ISSN : 2605-8359

Éditeur

Presses Universitaires de Provence

Édition imprimée

Date de publication : 15 juin 2015

Pagination : 191-205

ISBN : 978-2-85399-993-9

ISSN : 0751-4239

Référence électronique

Christiane Solte-Gresser, «Begabte Schwindlerinnen. Über die Inszenierungen der Lüge bei Ljudmila Ulickaja », Cahiers d'Études Germaniques [Online], 68 | 2015, Online erschienen am: 17 Dezember 2017, abgerufen am 01 Dezember 2020. URL : http://journals.openedition.org/ceg/1554 ; DOI : https:// doi.org/10.4000/ceg. 1554 


\title{
Begabte Schwindlerinnen. Über die Inszenierungen der Lüge bei Ljudmila Ulickaja ${ }^{1}$ Christiane SOLTE-GRESSER
}

Universität des Saarlandes

\begin{abstract}
Jeder Mensch erfindet sich früher oder später eine Geschichte, die er für sein Leben hält.

Max Frisch, Mein Name sei Gantenbein
\end{abstract}

\section{Lüge und Literatur}

\section{Literatur als Lüge}

„Kunst ist Magie, befreit von der Lüge, Wahrheit zu sein“. Mit diesem bekannten Satz aus den Minima Moralia ${ }^{2}$ bringt Adorno den merkwürdigen Status von Literatur zwischen Realität und Mythos auf den Punkt. Die Pointe ist freilich, dass der Wahrheitsanspruch selbst als Lüge entlarvt wird: Das Wahrhaftige der Kunst besteht gerade darin, dass sie nicht behauptet, wahr zu sein. Diese Wahrhaftigkeit kann nur in einer nicht diskursiven Sprache zum Vorschein gelangen und indem das Werk die Wirklichkeit gerade nicht widerspiegelt. ${ }^{3}$ Das Potenzial der Kunst besteht damit - anders gewendet - in der ästhetischen Form, durch die sich ihr kritischer Gehalt $^{4}$ und die Dimension des Möglichen ${ }^{5}$ ausdrücken.

1 Bei der Angabe von Namen und Titeln aus dem Russischen wird die wissenschaftliche Transliteration verwendet. Die Eigennamen der Romanfiguren werden der deutschen Übersetzung entsprechend übernommen.

2 Theodor W. Adorno, „In nuce“, in ders., Minima Moralia. Reflexionen aus dem beschädigten Leben [1951], Frankfurt a. M., Suhrkamp, 1994, S. 298.

3 Vgl. Theodor W. Adorno, Ästhetische Theorie, Frankfurt a. M., Suhrkamp, 1970, S. 251 und 419.

4 „Der Wahrheitsgehalt der Kunstwerke ist fusioniert mit ihrem kritischen“, ibid., S. 59.

5 „Dass aber die Kunstwerke da sind, deutet darauf, dass das Nichtseiende sein könnte. Die Wirklichkeit der Kunstwerke zeugt für die Möglichkeit des Möglichen“, ibid., S. 200. 
Beides, kritischer Anspruch und der Entwurf möglicher Welten durch ein Erzählen, mit dem die verlogene Wirklichkeit gebannt oder für einen Moment außer Kraft gesetzt wird, spielen in dem Roman, der Gegenstand dieses Artikels ist, eine entscheidende Rolle. Jedoch in anderer Weise als bei Adorno: Das Phänomen der Lüge wird nicht philosophisch durchdrungen, sondern fiktionalisiert in Szene gesetzt. ${ }^{6}$ Die Problematik ist damit auf eine diegetische bzw. eine intradiegetische Ebene verlagert: Das Erzähluniversum von Ljudmila Ulickajas episodenhaftem Roman Skvoznaja linija ${ }^{7}$ aus dem Jahr 2002 besteht aus zahlreichen lügenden Figuren, deren Geschichten von einer einzigen Zuhörerin, der Protagonistin des Romans, zusammengehalten werden: Es ist Shenja, die uns sämtliche Lügengeschichten im Hinblick auf ihre Motivation, Funktion, Tragweite und vor allem auf ihre Wirkung vor Augen führt.

Diese Tatsache führt zu zwei Thesen, die es zu überprüfen gilt: Erstens, die Lügen des Romans, die stets die Konstruktion oder Modifikation der individuellen Lebensgeschichte im autobiographischen Sprechakt betreffen, werden wie kleine literarische Erzählungen innerhalb der fiktionalen Welt inszeniert. ${ }^{8}$ Shenja liest als direkte Adressatin die dargebotenen Lügen wie Literatur, das heißt, sie lässt sich in ihren Bann ziehen, sie identifiziert sich mit ihren Figuren, sie kontextualisiert, deutet und bewertet die Geschichten. Damit wird sie, so meine zweite These, zu einer Rezipientin innerhalb der erzählten Welt. Der Roman inszeniert also nicht nur einen ganzen Reigen lügender Frauen, die sich um die Protagonistin herum gruppieren; er inszeniert auch geradezu mustergültig die tragende Rolle, die der Rezeption zukommt, wenn es darum geht, das Verhältnis von Lüge und Erzählung $\mathrm{zu}$ verstehen. Ich versuche daher, den Roman aus einer rezeptionsästhetischen Perspektive zu beleuchten. ${ }^{9}$

Reflexionen über Lüge und Wahrheit als literarische Phänomene bewegen sich zumeist in einem größeren fiktionstheoretischen Kontext. ${ }^{10}$ Monika Schmitz-Emans setzt sich in systematisch-typologischer Weise mit diesem Problem auseinander. ${ }^{11}$

6 Zum Erzählen (literatur)theoretischer Probleme und seinen Fiktionalisierungsstrategien vgl. Christiane Solte-Gresser/ Manfred Schmeling (Hrsg.), Raconter la théorie dans le roman, Würzburg, Königshausen und Neumann, 2015 (im Druck).

7 Ljudmila UlickaJa, Сквозная линия [Skvoznaja linija], Moskau, Ėksmo, 2002, dt: Die Lügen der Frauen (wörtl. „Durchgangslinie“ oder „durchgezogene Linie“), übersetzt von Ganna-Maria BraungardT, München, Hanser, 2003. Alle Angaben des Romans beziehen sich auf die deutschsprachige Ausgabe des Hanser-Verlages und werden abgekürzt zitiert mit LF und Seitenzahl.

8 Man könnte für diese erfundenen Ausflüchte vielleicht den Begriff ,petit récit“ verwenden, den Lyotard den „grands récits“ gegenüberstellt: „Le petit récit reste la forme par excellence que prend l'invention imaginative." Jean-François Lyotard, La Condition postmoderne, Paris, Minuit, 2005, S. $8 \mathrm{f}$.

9 Dabei wende ich allerdings nicht einen einzigen Ansatz konsequent an, sondern bemühe vielmehr - probeweise und ohne mich diesen detailliert widmen zu können - unterschiedliche Zugänge der Rezeptionstheorie, um Funktion und Wirkung der Lüge in Ulickajas Roman auf die Spur zu kommen.

${ }^{10}$ Vgl. Kurt Röttgers/ Monika Schmitz-Emans (Hrsg.), Dichter lügen, Essen, Die blaue Eule, 2001 (Philosophisch-literarische Reflexionen Bd. 3), S. 7-14 und bezogen auf das 20. und 21. Jahrhundert: Doren Wohlleben, „Die Poetologische Selbstbestimmung als Reaktion auf den Lügenverdacht“, in dies., Schwindel der Wahrheit. Ethik und Ästhetik der Lüge in Poetik-Vorlesungen und Romanen der Gegenwart, Freiburg i. Br., Rombach, 2005, S. 11-22.

11 Sie fasst verschiedene Thesen von Dichtern und Denkern zum Verhältnis zwischen Lüge und Literatur, die ihr zufolge auch immer verwandte Dichotomien wie Sein und Schein, Erfahrung und 
Auf der Grundlage von Schmitz-Emans' Typologie soll zunächst gefragt werden, wo und wie innerhalb dieser Kategorien des Lügens besonders das autobiographische Erzählen und die Dimension der Lügenrezeption berührt werden. Denn beides liefert für Die Lügen der Frauen einen wichtigen Schlüssel.

\section{Lüge als Autobiographie}

Das Erzählen der eigenen Lebensgeschichte bedarf eines beglaubigenden Zuhörers - und sei es nur, dass der Erzählende selbst an seine autobiographische Konstruktion glaubt. Die Grenzen zwischen Wahrheit und Lüge sind dabei notgedrungen fließend bzw. außer Kraft gesetzt. Denn das Leben lässt sich erzählend nicht abbilden. Ein kreatives Sprechen konstruiert die eigene Lebensgeschichte, indem es die Wirklichkeit verwandelt, verzerrt, verkehrt oder fiktional erweitert. ${ }^{12}$ Dieses Sprechen als Lüge zu identifizieren, setzt eine bestimmte Rezeptionshaltung voraus: nämlich dass vom Text eine Erkenntnis über das Leben und die Welt erwartet wird, die auf nachweisbaren Fakten beruht und daher rational überprüft werden kann. ${ }^{13}$ Willigt der Zuhörer jedoch in die spezifische Form literarischer Kommunikation ein - besteht also „Einverständnis darüber [...], dass die Aussagen der Dichtung keine Ist-Aussagen sind“"14, werden sie jenseits der Dichotomie von Lüge und Wahrheit verstanden. Eine solche Rezeptionshaltung gerät dann allerdings in Konflikt mit der klassischen Gattung der Autobiographie; zumindest dann, wenn man den Überlegungen Philippe Lejeunes zum autobiographischen Pakt folgen mag. ${ }^{15}$ In diesem Zusammenhang wird sich an Ulickajas Roman zweierlei zeigen: Die Haltung, mit der die Protagonistin Shenja die Geschichten aufnimmt, ist nicht statisch, sondern dynamisch. Ob sie diese als Lügen identifiziert oder als unterhaltsame Erzählung genießt, hängt von ihrer lebensweltlichen Erfahrung und augenblicklichen Situation ab, und das heißt vor allem auch, von den Geschichten, die sie zuvor gehört oder gelesen hat. Außerdem erweisen sich die Lügen hier allesamt als Lebenslügen im wörtlichen Sinne ${ }^{16}$ : Die Figuren machen nicht nur dem Gegenüber, sondern im Akt des Erzählens vor allem sich selbst etwas vor. ${ }^{17}$

Mario Vargas Llosa widmet sich in seinem Essay „Die Wahrheit der Lüge“ zwar

Traum, Realität und Fiktion einschließen, zu übergeordneten Kategorien zusammen, vgl. Monika Schmitz-Emans, „Zur Einführung“, in Röttgers/ Schmitz-Emans, Dichter lügen, S. 7-14.

12 Vgl. hierzu auch WoHLleBen, Schwindel der Wahrheit, S. 11.

13 Vgl. Schmitz-Emans, „Zur Einführung“, S. 8.

14 Ibid., S. 12.

15 Vgl. Philippe Lejeune, Le Pacte autobiographique, Paris, Seuil, 1975. Geht er diesen Akt ein, setzt der Leser bekanntlich voraus, dass Autor, Erzähler und erzähltes Subjekt identisch sind und der Erzähler um Authentizität oder zumindest um Aufrichtigkeit bemüht ist.

16 Zur Klärung des Begriffs, der Ibsens Theaterstück Die Wildente entstammt, vgl. Steffen DiETZSCH, Kleine Kulturgeschichte der Lüge, Leipzig, Reclam, 1998, S. 109.

$17 \mathrm{Vgl}$. „Les femmes évoquées par notre romancière rêvent leur vie et arrivent probablement à croire elles-mêmes à leurs chimères [...]. Nos héroïnes remplissent leur vie de ce qu'elles aimeraient y voir: l'amour, la poésie, et, pourquoi pas, le drame“ (Marie-José Selaudoux, „Mensonges de femmes de Ljudmila Oulitskaïa“, in La Critique parisienne, 67, 2012, S. 23-25, hier S. 24, http://www. lacritiqueparisienne.fr/67/oulitskaia.pdf [Stand: 31. Juli 2014]. 
nicht speziell dem autobiographischen Erzählen. ${ }^{18}$ Jedoch führt er ausdrücklich (und geradezu simplifizierend) vor Augen, wie eng Produktion und Rezeption fiktionaler Erzählungen miteinander verbunden sind. Sie treffen sich im Ungenügen sowohl der Erzähler als auch der Leser an der bestehenden Wirklichkeit:

[Erzählende Literatur] wird geschrieben und gelesen, damit die Menschen das Leben haben, mit dessen Nicht-Existenz sie sich nicht abfinden wollen. Im Keim jedes Romans [...] pulsiert ein Verlangen [...]. Romane werden nicht geschrieben, um das Leben zu erzählen, sondern um es zu verwandeln, indem man ihm etwas hinzufügt. ${ }^{19}$

In Ulickajas Roman fallen genau in diesem Verlangen Lügen-Produktion und Lügen-Rezeption zusammen: Für die Erzählerinnen wie die Zuhörerin greift die Lüge in die Wahrnehmung des eigenen Lebens ein, indem diesem sprechend oder hörend ein anderes gegenübergestellt wird; ein Leben - oder die Möglichkeit eines Lebens -, mit dessen Nicht-Existenz sich keine der beiden Seiten abfinden mag.

\section{Lüge und ,weibliches' Erzählen}

Auffällig ist indes, dass keine der bisher genannten Aussagen über Lüge und Erzählen aus einer geschlechtsspezifischen Perspektive erfolgt. Ulickaja aber formuliert durchaus explizite Aussagen zu diesem Problem. Geradezu essentialistisch stellt sie ihrem Roman Überlegungen voran, die fast schon als Gegenthese zu genderorientierten Denkrichtungen erscheinen, in denen Maskerade und Performanz von Geschlechterrollen als lügenhaftes Spiel entworfen werden:

Mon Dieu, comme elles [les femmes] peuvent mentir! [...] En passant, par mégarde, pour rien, avec ferveur, à l'improviste, en douce, à bâtons rompus, désespérément, sans la moindre raison. [...] Que de charme, que de talent, que de candeur et d'insolence, que d'inspiration créatrice et de panache! Il n'y a là ni calcul, ni espoir de profit, ni machinations... C'est juste une chanson, un conte, une devinette. Mais une devinette sans réponse. Chez les femmes, le mensonge est un phénomène de la nature, comme les bouleaux, le lait ou les frelons ${ }^{20}$.

Eine interessante Ergänzung hierzu liefert Ulickaja in einem Gespräch mit dem Spiegel, in dem sie vom Entstehungsprozess des Romans erzählt. Die weiblichen Lügen seien authentische, von ihr selbst gesammelte und ,zielten darauf ab, das eigene Leben auszuschmücken oder umzuschminken und der eigenen Biografie etwas hinzuzufügen“"21. Neben der durchaus irritierenden These, weibliches Lügen

${ }^{18}$ Er spricht vielmehr von der Macht der Lüge als einer „,eigentümliche[n] Wahrheit“ des Romans. Mario Vargas Llosa, Die Wahrheit der Lügen. Essays zur Literatur, übersetzt von Elke WeHr, Frankfurt a. M., Suhrkamp, 1994, S. 7f.

19 Ibid., S. 7.

${ }^{20}$ Dieses Vorwort wurde in die deutschsprachige Ausgabe nicht übernommen. Ich verwende daher die französische Übersetzung: Mensonges de femmes, übersetzt von Sophie BenECH, Paris, Gallimard, 2007.

${ }^{21}$ Rainer Traub, „Wir brauchen Provokateure“. Interview mit Ljudmila UlickaJA, in Der Spiegel, 41, 2003, S. 162-164, hier S. 162. Interessanterweise wird gerade diese Authentifizierungsstrategie in der russischen Verfilmung des Romans von Petr Stein aus dem Jahr 2005, in dem Ljudmila Ulickaja selbst mitspielt, wieder rückgängig gemacht. Der Spielfilm beginnt mit einer quasi dokumentarischen Rahmung: Die Autorin sitzt einer Frau gegenüber am Tisch, die ihr die Frage stellt, welche Haltung sie 
sei gewissermaßen ein naturgegebenes Gefühlsphänomen, fällt an Ulickajas Aussagen noch etwas anderes auf: Durchgängig wird hier in direkter oder indirekter Weise auf die Literatur Bezug genommen, sei es als Gattung oder als dichterisches Konstruktionsprinzip. ${ }^{22}$ Vor allem jedoch kommt hier eine ästhetische Haltung zum Vorschein, die sich sowohl auf die Produktion als auch die Rezeption von Lügengeschichten bezieht: Sie sind voll von kreativem Erfindungsgeist und sie beeindrucken, weil sie in einer talentierten Weise zur Darstellung gebracht werden. Damit liest sich die Aussage der Autorin als Loblied auf die lügnerische, ebenso wie auf die dichterische Begabung.

\section{Ulickajas Lügen der Frauen}

\section{Erzählstrukturen der Lüge}

Wie also lässt sich vor diesem Hintergrund das Lügen im Roman Skvoznaja linija verstehen? Für den Zusammenhang von Lüge, Weiblichkeit und Erzählung sind folgende Fragen besonders relevant, die in der Figur der Protagonistin gebündelt werden: Über die Rolle der binnenfiktionalen Rezipientin lässt sich erstens untersuchen, was die Erzählstruktur des Romans zum Phänomen der Lüge als Literatur beiträgt. Wie wird also das ,Lesen' der Lüge erzählt? Zweitens geht es um die selbstreflexive Dimension des Romans und damit um die Frage, inwiefern das Erzählen der Lüge zugleich über seine eigene Rezeption nachdenkt.

Die sechs Kapitel des Romans, die jeweils auf eine bestimmte Lügengeschichte konzentriert sind, spielen zu unterschiedlichen Zeiten, an unterschiedlichen Orten und erzählen gänzlich unterschiedliche Situationen. Über die Protagonistin Shenja werden sie lose miteinander verknüpft. Bei näherem Hinsehen ist allerdings durchaus eine Entwicklung zu erkennen; und zwar in dreifacher Weise. Erstens lässt sich anhand des Romans die Entwicklung von der Sowjetunion der Brežnev-Ära über die Perestrojka und Glasnost' bis in die postsowjetische Gegenwart rekonstruieren. Mit Blick auf das Gesamtwerk Ulickajas wird hier zwar vergleichsweise wenig gesellschaftspolitische Kritik geübt. ${ }^{23}$ Dennoch zeigt sich durchgängig: Die Figuren

selbst der Lüge gegenüber einnehme. Beide erzählen sich davon, dass sie selbst auch zu leidvollen Opfern der Lügen anderer geworden seien. Dabei bleibt offen, ob das Gegenüber von sich selbst in seiner Rolle als Interviewerin spricht - oder aber als literarische/ filmische Figur: Sie ist nämlich, wie sich einige Minuten später herausstellt, zugleich die Schauspielerin, welche die Protagonistin Shenja verkörpert, jene Figur, die in der Diegese tatsächlich mit zahlreichen Lügengeschichten konfrontiert wird. Damit spielt der Film von Beginn an mit den Grenzen zwischen Fiktion und Wirklichkeit, die in einer gemeinsamen Reflexion zweier Frauen am Beispiel der Lüge verhandelt werden.

${ }^{22}$ In einem anderen Zusammenhang werden solche Phantasiegeschichten auch ,nicht realisierte Träume“ genannt, vgl. hierzu Evgeniia Shcheglova, „The Unrealized Dream. The Prose of Liudmila Ulitskaia“, in Russian Studies in Literature, 49/1, 2013, S. 25-46, hier S. 25.

${ }^{23}$ Vgl. etwa Zelenyj šater (2010, dt: Das grüne Zelt), Danièl’ Štajn, perevodčik (2006, dt: Daniel Stein) oder Kazus Kukockogo (2000, dt: Reise in den siebten Himmel), drei Werke, die ein ausgesprochen kritisches politisches Potential besitzen. Anlässlich der Verleihung des Simone de Beauvoir Preises im April 2011 in Paris gibt die Autorin dennoch dem Privatleben gegenüber dem gesellschaftlichen den 
leiden unter den sozialen und politischen Bedingungen, sie versuchen, sich ihnen zu entwinden oder ihre individuellen Spielräume zu erweitern. ${ }^{24}$ Zweitens wird Shenjas Leben - mit zum Teil Jahrzehnte langen Ellipsen - chronologisch erzählt: vom Zeitpunkt der ersten Scheidung als junge Mutter und Doktorandin der Literaturwissenschaft über vielfältige Versuche im Liebes-, Berufs- und Alltagsleben $\mathrm{Fu}$ zu fassen, bis hin zu einem dramatischen Autounfall, der sie so schwer verletzt, dass sie gezwungen ist, sich zwischen einem mühseligen, nahezu aussichtlosen Überlebenskampf und Selbstmord zu entscheiden. ${ }^{25}$ Und drittens bewegt sich die Shenja vom Rand der Handlung immer mehr ins Zentrum hinein: Zunächst hört sie fast ausschließlich zu, dann greift sie als Reaktion auf die ihr zu Ohren gekommenen Geschichten in das Leben der anderen ein, später ist sie zunehmend in deren Beziehungen involviert, bis sie schließlich zur Hauptfigur wird, um die sich nun das Geschehen der anderen dreht.

Dementsprechend verringert sich der Anteil, d. h. Umfang und Dauer der Lügengeschichten kontinuierlich: Hatte die Lüge als Figurenrede einer Zufallsbekanntschaft, der Shenja mehr oder weniger passiv lauscht, zu Beginn noch fast das gesamte erste Kapitel eingenommen, werden solche Erzählungen im Verlauf des Romans immer schmaler, weniger autonom. Der fünfte Teil dezentriert sie, indem er sie auf zahlreiche Nebenfiguren verteilt, bevor die Lüge im Schlusskapitel schließlich nur noch ganz am Rande und eigentlich vor allem im übertragenen Sinne auftaucht.

Die Lebenswelt, in der Shenja die Lügengeschichten begegnen, ist auffällig weiblich inszeniert. Das Handlungsgeschehen wird in geradezu programmatischer Weise auf den Frauenalltag der Hauptfigur fokussiert. ${ }^{26}$ Aber auch die erlogenen Lebensgeschichten oder Lebensepisoden der anderen Frauenfiguren entwerfen ein auffällig weibliches Erzähluniversum: Es geht um Mutterschaft, die Rolle als kleine Schwester, um das leidenschaftliche Liebesleben eines frühreifen Mädchens, um die (fehlende) Anerkennung als Autorin und Professorin und die

Vorrang und formuliert damit eine grundsätzliche Haltung, derzufolge sie lediglich politische Texte schreibe, weil die Situation sie dazu zwinge (vgl. Ljudmila UlickAJA, „Meine Geschichte. Meine Geschichten. Die Autorin Ljudmila Ulickaja über ihr Leben und Werk“, in Xing. Ein Kulturmagazin, 23, 2012, S. 6-15, bes. S. 12). Vgl. hierzu auch Susanne SchOLL, „Die Menschen haben zu lachen begonnen. Ljudmila Ulickaja und die Politik“, in Xing. Ein Kulturmagazin, 23, 2012, S. 4. Zu einem ihrer dezidiert politischen Romane und seiner gesellschaftskritischen Dimension, vgl. Alla LATYNINA, ,,Soviet Power Has Killed Them All... 'Liudmila Ulitskaia's The Green Tent", in Russian Studies in Literature, 49/1, 2013, S. 8-24.

${ }^{24}$ Vgl. hierzu auch Ursula Sтоск in Rezensionen online. Literaturdatenbank des österreichischen Bibliothekswerks, http://www.biblio.at/rezensionen/ [Stand: 21. August 2014].

${ }^{25}$ Dieses letzte Kapitel tauchte in der russischen Erstausgabe des Romans zunächst nicht auf. Der Verlag hatte den offensichtlich unfertigen Text bereits veröffentlicht. Es ist jedoch derjenige Teil, der die anderen Geschichten zusammenführt und die Lügenproblematik von der mehr oder weniger harmlosen Schwindelei in eine existenzielle Dimension überführt.

${ }^{26}$ Das heißt, auf tägliche Verrichtungen und Besorgungen, Gespräche mit Freundinnen und Bekannten, Organisation des Familienlebens und einen beruflichen Auftrag, den sie ausdrücklich erhält, weil sie eine Frau ist. Zum Alltag im Werk von Ulickaja vgl. auch Christine DAUM, ,,Russische Autorinnen: Im Osten nichts Neues?“, in Emma, 5, September/ Oktober 2003, http://www.emma.de/artikel/russischeautorinnen-im-osten-nichts-neues-265373 [Stand: 10. Februar 2014]. 
Aufstiegsversuche von russischen Prostituierten in der Schweiz. In jedem Kapitel wird deutlich, dass dieses Leben ausgesprochen mühselig und von vielen Seiten durch die spezifisch sowjetischen bzw. postsowjetischen Bedingungen eingeschränkt ist. Die Lügengeschichten lassen sich als Ausflüchte aus den Grenzen dieses gesellschaftspolitisch beengten Alltags verstehen. ${ }^{27}$

Wie also werden diese einzelnen Lügengeschichten als Literatur inszeniert und vor allem rezipiert? Ungeachtet der genannten Entwicklung im Roman bleibt der Modus der Erzählung im Laufe des Geschehens konstant. Die heterodiegetische Erzählinstanz rückt ausgesprochen nah an das Erleben der Protagonistin heran. Shenja bildet durchgängig die intern fokalisierte Wahrnehmungs- und Rezeptionsperspektive. Hieraus ergibt sich, dass die Erzählstimme selbst die Figurengeschichten nicht bewertet oder über Lüge und Wahrheit entscheidet. Es finden sich stattdessen allgemeinere Begriffe wie Sprechen, Erzählen, Gespräch, Geschwätz, Bericht, Geplauder oder Unterhaltung. ${ }^{28}$ Die Klassifizierung als Lüge, Wahrheit, Schwindelei, Erfindung oder Erdichtung erfolgt also - während oder im Anschluss an die Figurenrede - einzig durch die Einschätzung der Zuhörerin.

\section{Rezeption der Lügenepisoden}

Im ersten Kapitel trifft Shenja in ihrem Urlaubsdomizil auf eine geheimnisvolle Engländerin, die ihr die Tragödie ihrer Mutterschaft erzählt. Eingeleitet wird sie mit der Bemerkung, dieses Geheimnis noch niemals zuvor erzählt und nun ausgerechnet Shenja zur Vertrauten auserkoren zu haben; also einer klassischen captatio benevolentiae. ${ }^{29}$ Ireen, verheiratet mit einem Mann, dessen Großvater in eine spektakuläre Spionagegeschichte involviert war, verliert in der tragischen Verkettung unglückseliger Umstände ihre ersten vier Kinder. Erst das fünfte schließlich überlebt die Jahre bis zu jenem Urlaubsabend, der die Erzählsituation abgibt. Die Handlung dieser Geschichte wird in ihrem unabänderlich auf die Katastrophe zusteuernden Verlauf - ganz wie ein tragisches Bühnenstück - mehrfach durch retardierende Momente durchbrochen. Shenja rezipiert sie denn in der Tat

\footnotetext{
${ }^{27}$ Vgl. hierzu auch Christa EBERT, ,,,Die Glücklichen'. Familie als Gemeinschaftsutopie in der Prosa von Ljudmila Ulitzkaja“, in dies./ Brigitte SÄNDIG (Hrsg.), Ideen und Bilder von Gemeinschaftlichkeit in Ost und West, Frankfurt a. M. [u. a.], Lang, 2008, S. 143-157. Ebert gelangt - allerdings auf andere Werke Ulickajas bezogen - zu dem Schluss, die Texte seien „Gegenerzählungen zum offiziellen sowjetischen Geschichtsnarrativ“ (ibid., S. 156).

${ }^{28}$ Vgl. O. N. ČISTJAKova, „Lož’ kak kommunikativnaja strategija jazykovoj ličnosti (po povesti L. Ulickoj ,Skvoznaja linija‘ [Die Lüge als kommunikative Strategie der sprachlichen Persönlichkeit (anhand von L. Ulickajas Kurzroman ,Skvoznaja linija')]“, in N. A Andramonova (Hrsg.), Russkaja i sopostavitel'naja filologija, Kazan', Izdatel'stvo Kazanskogo Gosudarstvennogo universiteta, 2006, S. 293-297, bes. S. 292 f. Für die zahlreichen hilfreichen Hinweise zur russischen Sprache und zum slawistischen Gesamtkontext des Romans danke ich herzlich Marco Klüh.

${ }^{29}$ Interessanterweise ist dieses Drama quasi mythologisch gerahmt: Ireen beginnt und endet mit der mysteriösen Prophezeiung durch eine Wahrsagerin: „Und du fängst mit dem fünften [Kind, C. S] an. Denk daran: mit dem fünften“ (LF 15). Auf dem Hintergrund dieses Fluches kann sich die Geschichte nur noch als Realisierung der vorhergesagten Katastrophe entspinnen.
} 
auch wie eine aristotelische Tragödie ${ }^{30}$ : Sie leidet mit den Figuren und lässt sich von der betörenden Schönheit der Tochter Diana berauschen. Sie schauert und erbebt angesichts der schrecklichen Schicksalsschläge und ist beeindruckt von der Würde, mit der die Erzählerin diese erträgt. ${ }^{31}$

In diesem Zuhören liegt eine doppelte Bewegung: Einerseits entführt diese Geschichte, die in der Tat ein beachtliches eskapistisches Potenzial birgt, sie aus ihrem eigenen Leben, so dass sie dieses aus der Distanz heraus wahrnimmt ${ }^{32}$ : ,[Sie] genoss zugleich die [...] kurzzeitige Freiheit von den ungelösten wichtigen - waren sie wirklich so wichtig? - Problemen ihres Lebens" (LF 16). ${ }^{33}$ Andererseits versteht die Zuhörerin das Dargebotene fast ausschließlich im Hinblick auf das, was Norman N. Holland aus psychoanalytischer Rezeptionsperspektive als das eigene identity theme bezeichnet hat: eine identifikatorische Lektüre, derzufolge jeder Leser in einer fiktionalen Geschichte vor allem sein eigenes Lebensthema und die damit verbundenen existenziellen Fragen wiederfindet. ${ }^{34}$ Was auch immer sie hört, sie versteht und bewertet es in Relation zu ihrem problematischen Liebesleben, das sich wie ein roter Faden durch den Roman zieht. ${ }^{35}$

Wie sehr Shenja beim Zuhören der Macht des „als ob“ erlegen ist, macht die Auflösung des Kapitels deutlich: die Entlarvung der autobiographischen Erzählung als Lüge. Konstruiert ist diese durch die Ankunft eines neuen, mit Ireen befreundeten Pensionsgastes, der die „Gegengeschichte, die wahre“ erzählt (LF 33) - wobei sich herausstellt, dass ausgerechnet das unwahrscheinlichste Detail, die verrückte Spionageaffäre, das einzig nicht Erlogene war. Die Lüge wird also durch eine glaubwürdigere Figur identifiziert - so dass im Nachhinein auch die vielen Signale der Unzuverlässigkeit des Erzählten, wie Trunkenheit, Wahnsinn,

${ }^{30}$ Vgl. Selaudoux, „Mensonges de femmes“, S. 24, die umgekehrt zeigt, dass sich die Sprechende als Heldin einer Tragödie inszeniert.

31 „Shenja hörte mit offenem Mund zu [...] und wurde immer berauschter, nicht mehr vom Wein [...], sondern weil sie so hingerissen und begeistert war“ (LF 9); „dieser potentielle wunderschöne Gesang erschütterte Shenja, und die schmerzhafte Bitterkeit dieser Geschichte brach nun doch in Tränenströmen aus ihr heraus“ (LF 21); „Shenja betrachtete sie mit stillem Neid: was für eine Biographie!“ (LF 18). Man könnte hier geradezu systematisch das Interaktionsmuster zwischen Figur/ Erzähler und Leser/ in übertragen, das Hans Robert Jauß für den Rezeptionsprozess entworfen hat: Er verläuft assoziativ, admirativ, sympathetisch, kathartisch und ironisch (vgl. Hans Robert JAuss, Ästhetische Erfahrung und literarische Hermeneutik, Frankfurt a. M., Suhrkamp, 1982, S. 252).

${ }^{32}$ Beispielsweise, indem sie das Erzählte vor sich sieht ,wie im Film“ (LF 21).

33 Vgl. auch LF 11.

34 Vgl. Norman Holland, The Dynamics of Literary Response, New York, Oxford University Press, 1968 und Five Readers Reading, New Haven, Yale University Press, 1975. Hervorzuheben wäre für den Zusammenhang unseres Romans u. a. der Hinweis auf den mündlichen Ursprung der zur Identifikation einladenden Geschichte und dass der Rezeptionsprozess umso intensiver verläuft, je mehr das Zuhören im Ruhezustand erfolgt. Mieke BAL (Narratology. Introduction to the Theory of Narrative, Toronto, University of Toronto Press, 1985, bes. S. 153-163) hat gezeigt, dass besonders die Fokalisierung auf eine bestimmte Figur und der Gebrauch der erlebten Rede den Identifikationsprozess in Gang setzen.

${ }^{35}$ Diese Rezeptionshaltung ist übrigens ausdrücklich in einen intertextuellen Rahmen, nämlich ihre Lektüre von Anna Karenina, eingebettet: ,[...] Shenja [...] [las] ,Anna Karenina', um einige Ereignisse ihres zerbröckelnden Privatlebens zu vergleichen mit dem echten Drama einer richtigen Frau“ (LF 8); ,[...] Shenja [...] [konnte] sich nicht vom Schicksal der rothaarigen Engländerin losreißen, gegen das Anna Kareninas altmodischer Selbstmord verblasste und wirkte wie die Laune einer überdrehten feinen Dame“(LF 31). 
Prahlerei ins Auge springen müssen. Die (Über-)Reaktion Shenjas kann nur als Antwort auf ihre identifikatorische Lektüre verstanden werden: Sie weint, bricht in hysterisches Lachen aus und untersucht des Nachts die „seelische Wunde“, die Ireens Lüge hinterlassen hat; einen „Phantomschmerz“ von „sinnlos verausgabtem Mitgefühl mit den nichtexistenten, genial erfundenen und unmenschlich getöteten Kindern“ (LF 34). Zugleich aber ist sie beschämt darüber, dass die Geschichte so gut funktioniert hat: ,[...] sie [...] fühlte [sich] wie ein dummes Kaninchen, das einem sinnlosen Experiment unterzogen worden war" (LF 35). Festzuhalten bleibt: Die Empörung ist an keiner Stelle eine moralische; sie bezieht sich weniger auf einen etwaigen Vertrauensmissbrauch durch die Lügnerin, als vielmehr auf die gelungene Grenzüberschreitung zwischen Realität und Fiktion. ${ }^{36}$

Das zweite Romankapitel lässt sich nur auf der Folie des ersten verstehen - es ist dessen Wiederholung und Umkehrung zugleich: Wieder wird eine Feriensituation mit entfernten Bekannten beschrieben. Wieder ist die Protagonistin, eine Scheidung später, damit beschäftigt, „zu lesen und über ihr mißglücktes Liebesleben nachzudenken“ (LF 39, vgl. auch LF 42). Wieder wird eine Lügengeschichte erzählt, und wieder klärt eine hinzukommende Figur die Zuhörerin über deren Wahrheitsstatus auf. So verbringt die zehnjährige Nadja ihre Ferien damit, Shenja die aufregendsten Begebenheiten aus ihrem bisherigen Kinderleben zu erzählen. Diese sind in auffälliger Weise an Mustern des Kriminal- und Abenteuerromans oder der trivial-kitschigen Aufstiegsgeschichte orientiert: die naive Idealisierung ihres heldenhaften Bruders Jurotschka, die Aufklärung eines Mordfalls mit anschließender Belohnung, die Auswahl zu einer Spanienreise für ein russisches Filmprojekt, in dem sie die Hauptrolle spielt, und schließlich gar die Landung eines Ufos, die Nadja als einzige beobachtet haben will. Shenja ist zunächst versucht, die Aufschneiderei zu unterbinden, schiebt ihre moralischen Bedenken aber in dem Moment zur Seite, in dem sie sich entscheidet, das Erzählte wie eine unterhaltsame Ferienlektüre zu rezipieren.

Sie spielten Schafskopf , um eine Geschichte' [...]. Wer verlor, mußte eine Geschichte erzählen - lustig, gruselig, komisch, je nach Wunsch der anderen. Nadja schwadronierte gerade, phantasievoll, und ohne die geringste Glaubwürdigkeit, wie sie letzten Sommer zu Filmaufnahmen in Spanien war, ein Pferd bekam, das zuvor bei Stierkämpfen aufgetreten, aber wegen nervlicher Zerrüttung zum Filmstudio versetzt worden war. (LF 43)

Die Abenteuererzählungen des Mädchens funktionieren also wie die Geschichten von Scheherazade: Das Mädchen darf so lange am Urlaubsort verweilen, wie sie die Gastgeberin zu unterhalten weiß. Indem sie die Zuhörerin vortrefflich bei Laune hält, wird eine ethisch-moralische Entscheidung über Lüge oder Wahrheit hinfällig. ${ }^{37}$

Shenjas Haltung als Zuhörerin erklärt sich aus einem ganz bestimmten

${ }^{36}$ Diese besitzt eine so nachhaltige Wirkung, dass Shenja auch wider besseres Wissen die ,geniale Erfindung“ noch Wochen später mit der Wirklichkeit verwechselt: „Doch hin und wieder durchzuckte es Shenja: Ireen! Warum hatte sie sie [ihre Kinder, C. S.] alle getötet?“ (LF 36).

${ }^{37}$ Dies schließt allerdings nicht eine geschlechtsspezifische Reflexion über das Lügen von Mädchen und Jungen aus: „Verblüffend [...]. Jungen schwindeln doch auch. Aber immer zu einem bestimmten Zweck: um einer Strafe zu entgehen, um eine Untat zu verheimlichen.“(LF 45). 
Erwartungshorizont. ${ }^{38} \mathrm{Nicht} \mathrm{nur} \mathrm{die} \mathrm{Erinnerung} \mathrm{an} \mathrm{Ireens} \mathrm{Mutterschaftstragödie,} \mathrm{auch}$ ihre umfassenden Lektüreerfahrungen als promovierte Literaturwissenschaftlerin bieten ihr die Möglichkeit, das Gehörte vergleichend einzuschätzen und als größenwahnsinnige Phantasien zu erkennen. Beides, lebensweltliche und literarische Muster, liefern also die Folie für die Rezeption der Erzählungen. Dies macht Shenja zur perfekten Geschichtenadressatin: Sie beurteilt das Gehörte nun nicht mehr nach der Überprüfbarkeit des Inhalts, sondern nach der Originalität der Darbietung:

[Nadja] schwindelte [...] doch sehr amüsant und irgendwie außergewöhnlich. (LF 44)

Shenjas Gereiztheit klang [durch die kunstvollen Erzählungen] ab, sie lachte. (LF 40)

Shenja staunte nur, wie begabt die kleine Schwindlerin war. (LF 45)

Die Pointe dieses Kapitels besteht nun freilich darin, dass sich nach einer Befragung der Mutter sämtliche Erlebnisse des Mädchens als wahr erweisen. Einzig und allein das, was Shenja - und wir Leser - als wirklich angenommen hatten, die Existenz des famosen großen Bruders, war eine Schwindelei, mit der sich das Kind aus seiner Einsamkeit herausgelogen hat.

Das Kapitel „Ende der Geschichte“ ist vor allem aufgrund seiner Auflösung interessant. Hier stilisiert sich eine Dreizehnjährige lolitahaft zur Geliebten ihres Onkels - bezeichnenderweise trifft sie sich mit ihm stets, indem sie „eine Doppelstunde Literatur“" schwänzt (LF 57). Hatte Shenja im Kapitel zuvor durch ihre ästhetische Haltung die Intention unterdrückt, ein Lügen zu verbieten, das gar keines war, versucht sie hier umgekehrt, eine moralische Haltung einzunehmen und eine Handlung zu beeinflussen, die sich schließlich als erfunden herausstellt. Allerdings wirkt diese Fiktion durchaus auf die Wirklichkeit ein - oder zumindest auf die Wahrnehmung dieser Wirklichkeit durch die Rezipientin: Die äußerlichen Veränderungen des Mädchens führt sie auf Liebeserfahrungen zurück, die rein fiktiv sind (LF 58). Moralisch entrüstet, sucht sie das direkte Gespräch mit dem Onkel. Dieser scheint die skandalösen Vorwürfe zunächst zu bestätigen. Doch nach und nach wird Shenja - und zugleich dem Leser - klar, dass das Gegenüber von einer anderen Liebesgeschichte erzählt. Die Affäre des Mädchens entpuppt sich damit rückblickend als pubertäre Phantasie. Unterhaltsam sind diese Passagen deshalb, weil der Prozess des ,Lese'-Verstehens schrittweise vor Augen geführt wird. ${ }^{39}$ Shenja ordnet die Rede des Onkels vor einem ganz bestimmten Erwartungshorizont ein, der die Rezeption lenkt. „Arkadi ist aufrichtig erstaunt. Er markiert den Ahnungslosen“ (LF 63). Vor allem aber füllt sie die Unbestimmtheitsstellen seiner Erzählung mit ihrem eigenen Wissen. ${ }^{40}$ Sie stellt Analogien her und zieht Schlussfolgerungen („Das dritte Jahr? Er hat sich also mit einer Zehnjährigen eingelassen? Und redet darüber so alltäglich... “, LF 63), so dass sich ein grotesker dialogue de sourds

38 Vgl. Hans Robert Jauss, Literaturgeschichte als Provokation, Frankfurt a. M., Suhrkamp, 1970, S. 144-207, v. a. S. 177-194.

39 „Da begreift Shenja, daß alles nicht so einfach ist, daß hinter diesen Worten mehr steht, als sie weiß. Arkadi wirkt teils schuldbewußt, teils aber auch leidend.“(LF 62).

${ }^{40} \mathrm{Vgl}$. hierzu Wolfgang Isers Theorie der Leerstellen im literarischen Text in: Der Akt des Lesens. Theorie ästhetischer Wirkung, Frankfurt a. M., Suhrkamp, 1976 und Wolfgang IsER, Die Appellstruktur der Texte. Unbestimmtheit als Wirkungsbedingung literarischer Prosa, Konstanz, Universitätsverlag, 1970. 
entspinnt. Dieser führt geradezu mustergültig vor Augen, wie eine Geschichte organisiert ist. Sie basiert stets auf Selektionen, funktioniert als Appell und ist damit auf einen Rezipienten angewiesen, der die Leerstellen schließt, z. B. indem er kausale Verknüpfungen herstellt. So entstehen in diesem Kapitel durch das aktive Zuhören der Protagonistin drei verschiedene, gleichermaßen kohärent erzählte Liebesgeschichten: eine erlogene, eine missverstandene und eine wahre.

Das vierte Kapitel verlegt die Rezeptionsproblematik noch sehr viel offensichtlicher in die Diegese selbst hinein; insofern nämlich, als es hier tatsächlich um Literaturwissenschaft geht. Eine Literaturprofessorin im Ruhestand bringt einer bildungsfernen, aber literaturhungrigen jungen Studentin die russische Lyrik näher. Hierbei vermischen sich die Grenzen zwischen dem (Re-)Zitieren fremder Texte und ihren eigenen Worten zusehends. $\mathrm{Ob}$ die naive Mascha nun aus reinem Wunschdenken der bewunderten Professorin die Urheberschaft der fremden Gedichte zuschreibt oder ob diese deren Unerfahrenheit ausnützt und selbst sich als Lyrikerin und Urheberin der Gedichte ausgibt, bleibt aufgrund der geringen Distanz zwischen Erzählstimme und Figurenwahrnehmung in der Schwebe: „Nach und nach stellte sich heraus, daß auch Anna Weniaminowna selbst eine Dichterin war.“(LF 69).

Auf einer Meta-Ebene aber setzen sich beide Gesprächspartnerinnen eindeutig mit diesem Problem auseinander. Mascha erhält von der alten Frau eine Art PrivatVorlesung über Autorschaft und Intertextualität, der sie entnimmt „daß der modische Autor nicht vom Mond gefallen war, daß er Vorläufer gehabt hatte, von denen sie nichts ahnte, und daß überhaupt jedes Buch sich auf etwas stützte, das zuvor schon gesagt und geschrieben worden war“ (LF 70). Als nun die Professorin stirbt, beginnt Macha während der Totenfeier, die „nie veröffentlicht[en]“ Werke der „wunderbar[en] Dichterin“"vorzutragen (LF 76). Weil es sich aber um Texte handelt, die alle Anwesenden sofort als Lyrik von Vološin oder Cvetaeva identifizieren, verurteilen sie nicht die professorale Hochstaplerin, sondern diejenige, die dem Schwindel/Missverständnis aufgesessen ist. ${ }^{41}$ Literaturwissenschaftlich-professionelles Lesen verhindert hier gerade das Erkennen der Wahrheit: Alle Trauergäste sind sich aufgrund ihrer Lektüreerfahrungen und des Bildes, das sie sich von einer Professorin machen, sicher, „daß dieses Ingenieursmädchen mit dem groben Gesicht und den dicken Beinen eine Vollidiotin war, die Anna Weniaminowna falsch verstanden und ihr etwas angedichtet hatte, was der kultivierten Professorin nie in den Sinn gekommen wäre“ (LF 79, 80). In diesem Kapitel vermischen sich also ästhetische und moralisch-lebensweltliche Rezeption. Dies bewirkt, dass die Lüge schließlich einer Figur zugeschrieben wird, die ihr selbst durch naiv-bewunderndes Zuhören zum Opfer gefallen war. ${ }^{42}$

${ }^{41}$ Zugleich wird hier die geradezu klassische Geschichte einer scheiternden Professorin erzählt; auch wenn dieses erst posthum und allein aus der Perspektive der Protagonistin erkennbar wird: Die Wissenschaft, mit der sich Anna Weniaminowna beschäftigt, ist aufs Engste verstrickt mit dem lebensweltlichen Problem, das sie zu Fall bringt - und dieser Fall kommt durch die narzisstische Kluft zwischen außen und innen, dem öffentlichen Ruf und der inneren Seelengröße, zustande. Vgl. hierzu: Christiane Solte-Gresser, „Der Sturz aus dem Elfenbeinturm. Komparatistische Begegnungen mit Professorenfiguren der Gegenwart", in Comparatio. Zeitschrift für Vergleichende Literaturwissenschaft, 4/1, 2012, S. 25-52.

42 Shenja ist die einzige, die am Schluss versucht, sich eine bewusste Lüge seitens ihrer Doktormutter vorzustellen. Auch wenn sie letztlich keine eindeutige Motivation erkennen kann, so scheint mit ihren 
Von der Literatur auf den Film übertragen wird die Lügen- und Rezeptionsproblematik schließlich im fünften Kapitel, dem merkwürdigsten des Romans. Hier stehen sich zwei verschiedene Zugänge bzw. Genres gegenüber, eine Lebensgeschichte zu erzählen: Der Dokumentarfilm und die Hollywood-Romanze. Verbunden werden sie über die Frage, wer auf welche Weise das Drehbuch verfasst. Shenja wird von einem dubiosen Regisseur beauftragt, in der Schweiz russische Prostituierte für das Fernsehen zu interviewen. Was der Filmemacher sucht, ist ebenso eindeutig wie hoffnungslos: ,,ich brauche [...] die Geschichte jedes einzelnen Mädchens. Die wahre Geschichte.“ (LF 84) Das Irritierende dieser Episode entsteht durch die zahllosen Unzuverlässigkeits-Signale. ${ }^{43}$ Das einzig Verlässliche scheinen die Daten und Fakten zu sein, welche die Interviewpartnerinnen preisgeben. Je mehr die Protagonistin jedoch an solchen Lebensgeschichten zusammen trägt, desto deutlicher kristallisiert sich ein übergeordnetes Muster heraus, an dem sich sämtliche Lebensentwürfe orientieren. Glaubt man als Leserin zunächst noch an merkwürdige Analogien und zufällige Übereinstimmungen, so werden die Erzählungen zunehmend schablonenhaft. Alle Frauen konstruieren ihre Geschichte nach einer Art Meta-Skript ${ }^{44}$, aus dem Shenja schließlich „eine typische Struktur heraus[filtert]“ (LF 102). Sie begegnet stets dem Aufstiegsmärchen vom ehemals wohlbehüteten und dann durch den Stiefvater in die Prostitution getriebenen Mädchen, das nach zahlreichen tragischen Verstrickungen schließlich einen reichen Schweizer Banker heiratet. Dies veranlasst den Regisseur letztlich dazu, den „Dokumentarquatsch“ aufzugeben und stattdessen einen Spielfilm zu drehen - „eine Art russische Lolita“, „das ist genial! Oscarreif! Mit Nathalie Portman in der Hauptrolle! [...] das wird wie Dostojewski!““(LF 104).

Der lügnerische Selbstbetrug in diesem Kapitel spielt sich auf mehreren Ebenen ab. Nicht nur verkennt der Regisseur, dass sein Spielfilmprojekt keineswegs genial ist, sondern zwölf Jahre nach Pretty Woman abgedroschener kaum sein könnte. Das Beklemmende ist, dass vor allem auch die erzählenden Frauen selbst an die Originalität ihres Lebensentwurfs glauben. Wie Emma Bovary ihr Leben auf der Grundlage identifikatorischer Lektüren nach literarischem Vorbild entworfen und ihr eigenes Gefühlsleben am Muster des Ritterromans oder der romantischen Liebesgeschichte ausgerichtet hatte ${ }^{45}$, so wird hier die „Stadtromanze“, das

Überlegungen das Verlangen auf, das Vargas Llosa Produzenten wie Rezipienten von Geschichten zugeschrieben hatte: Um mit einer Lügengeschichte das eigene Leben fiktiv zu erweitern und wenigstens ein Mal wie ein „große[r] Dichter“ oder ein „nichtige[r] Schreiberling“ „, in den willigen, einfältigen Herzen seiner Zuhörer Emotionen [...] [zu] weck[en]“ (LF 80), braucht es jedoch genau solche gutwilligen, empathischen und literaturaffinen Zuhörerinnen wie Shenja und Mascha es sind.

${ }^{43}$ Shenja kommt sich auf ihrer Reise vor wie im Traum, ihre Wahrnehmung ist durch Alkoholkonsum massiv eingeschränkt, sie erlebt das Geschehen wie einen Film und der Regisseur verhält sich auf rätselhafte, undurchsichtige Weise.

${ }^{44}$ Im Text ist auch von „Roman“ oder „Märchen“ die Rede, vgl. LF 95 und 98.

${ }^{45}$ Bereits pointiert beleuchtet von Jules de GaUthieR, Le Bovarysme. La psychologie dans l'œuvre de Flaubert, Charleston, SC, Nabu Press, 2000. Vgl. mit methodisch überzeugenderem Ansatz Claudia JÜNKE, Die Polyphonie der Diskurse. Formen narrativer Sprach- und Bewusstseinskritik in Flauberts Madame Bovary und L'Éducation sentimentale, Würzburg, Königshausen und Neumann, 2003. 
„Melodram für Arme“ (LF 109), zur Authentizität beanspruchenden (Auto-) Biographie. ${ }^{46}$ Aber die Erzählerinnen schreiben auch selbst an diesem Skript weiter. Sie tragen ihre Geschichte etwa höchst unterschiedlich vor oder bewerten sie vollkommen gegensätzlich, ohne den plot zu verändern. ${ }^{47}$ Damit zeigt sich zweierlei: Discours und narration machen diese, wie jede Geschichte erst zu dem, was sie ist. Und die Grenzen zwischen Fiktion und Realität werden hier ein weiteres Mal unscharf. Die Dichotomie zwischen Wahrheit und Lüge greift somit auch für dieses Kapitel zu kurz.

\section{Fazit: Lügenhaftes Leben?}

Das Schlusskapitel des Romans ist nicht nur das längste, es fällt auch in anderer Hinsicht aus dem Rahmen. Lügen im klassischen Sinne werden hier keine erzählt. Über den Begriff bzw. den Vorwurf der Lüge wird jedoch durchaus die Differenz zwischen wahrhaftiger und unaufrichtiger Lebenserzählung ausgelotet. Es geht um den Unterschied zwischen unpersönlicher Rede im Namen allgemeiner Werte und Normen und einer Rede, für die die Sprecherin mit ihrer ganzen Person einsteht, der sie also innerhalb ihrer eigenen Lebenswirklichkeit Glaubwürdigkeit verleiht. Der Lügenvorwurf erfährt damit eine gewissermaßen existenzialistische Wendung. Ihm kommt die ethische Funktion zu, die Kluft zwischen theoretischem Sprechen und praktischem Handeln zu befragen.

Literatur spielt hier nur noch am Rande eine Rolle. ${ }^{48}$ Nach einem dramatischen Auto-Unfall versuchen Verwandte und Freunde, die dahinsiechende Protagonistin ins Leben zurück zu holen. Jedoch weder wissenschaftlich-rationale noch religiöse, metaphysische grands récits können Shenja erreichen; allein für den Lügenvorwurf einer Freundin ist sie empfänglich. Dieser Vorwurf impliziert die Aufforderung, Shenja möge ihre früher geäußerten Ansichten und Überzeugungen nicht Lügen strafen. Erzählte doch die erste Hälfte des Kapitels ${ }^{49}$ von den unzähligen Bemühungen der Protagonistin, die Freundin nach deren Schlaganfall zum Weiterleben zu

${ }^{46}$ Zur rezeptionstheoretischen Differenzierung in triviale und literarisch-ästhetische Texte vgl. die (empirische) Studie von Katrin TrePte, Zwei Leserinnen lesen. Studien zur Identifikation bei der Literaturrezeption, Norderstedt, Books on Demand, 2004, v. a. S. 32-38. Die Frage nach Gemeinsamkeiten und Unterschieden der Rezeption zwischen professionellen und nicht professionellen Leserinnen ist Gegenstand der Studie von Martin SexL, Sophokles, Shakespeare und Tolstoi im Krankenhaus. Krankenpflegerinnen lesen literarische Texte, Wien/ Innsbruck, Studienverlag, 2006.

47 So wird beispielsweise der vermeintliche Aufstieg von Ljuda, der Heldenfigur aller anderen Prosituierten, von dieser selbst als gnadenloses Scheitern erzählt (LF 108 und 109).

${ }^{48}$ Shenja hat einen Verlag gegründet und ist auf dem Weg zur Frankfurter Buchmesse, als sich der Autounfall ereignet, bei dem sie fast ums Leben kommt. Was sie bis zum Zeitpunkt des Unfalls notgedrungen rezipiert hatte, waren die nervtötenden Geschichten ihrer aufdringlichen Freundin. Diese sind zwar von der inzwischen mehrfachen Großmutter Shenja aufgrund ihrer früheren Erfahrungen immer auch dem prinzipiellen Lügenverdacht ausgesetzt. „Ein vager Verdacht durchzuckte Shenja - sie hatte schon einmal eine Geschichte von toten Kindern gehört“ (LF 117). An keiner Stelle jedoch findet sich eine Bestätigung dafür.

${ }^{49}$ Auch dieser letzte Teil ist also erzähltechnisch durch die Gleichzeitigkeit von Wiederholung und Umkehrung strukturiert. 
motivieren. Shenjas unermüdlich geäußerte Devise, den Mut nicht zu verlieren oder sich Mühe zu geben, erweist sich aus der Perspektive des Gegenübers rückblickend als unaufrichtig: Der Vorwurf, die Worte seien womöglich nur dahin gesagt worden, nicht ernst gemeint und vor allem nur für andere, nicht aber für Shenja selbst gültig, betrifft die Kluft zwischen einem scheinheilig geäußertem Lebensoptimismus und tatsächlicher Lebensmüdigkeit: „Das heißt also, du hast mir was vorgemacht, ja? [...] Das war also nur Schwindel, als Du gesagt hast, ich muß wieder aufstehen, muß meinen Arm trainieren, alles neu lernen? [...] du bist eine Lügnerin und Verräterin!“ (LF 151).

Damit erweist sich die weibliche Lüge zum Ende des Romans hin, entgegen der Behauptung Ulickajas im Vorwort, doch als strategisch: Sie wird von einer Figur bewusst eingesetzt, um das Verhältnis von Lüge, Wahrheit und Erzählung auf das Verhältnis von Lebensmotto und Lebenspraxis zu überführen, dem einzigen Bereich, in dem ethische und moralische Kategorien Sinn machen. Man mag den Schluss naiv oder pathetisch finden: Es ist jedenfalls die Einsicht in die Verlogenheit der eigenen Rede, die Shenja schließlich dazu bringt, sich dem wirklichen Leben in all seiner Verlogenheit zu stellen.

Versteht man den Originaltitel des Romans Durchgehende [durchgezogene] Linie als eine poetologische Aussage zur Erzählstruktur des Textes, versucht man also eine Verbindung zu ziehen von den ersten Lügengeschichten bis zur letzten, so ergäbe sich in der Tat eine Linie: Die Rezeptionshaltung der Protagonistin verläuft vom identifikatorischen über das moralische, das literaturwissenschaftliche und das journalistische Lesen bis hin zum bewussten Um- oder Weiterschreiben der eigenen Lebensgeschichte. Auf die Produktion der Lüge übertragen, entsteht damit eine Linie von der zwecklosen, rein auf Dramatik und Unterhaltung angelegten - der gewissermaßen literarischen - Lüge über die Verzweiflungslüge, die ein Leben in Gewalt und Erniedrigung mit einer happy ending story überschreibt, bis hin zur Lüge als ethischem Apell ${ }^{50}$, als Überlebensstrategie ${ }^{51}$ : Wahrheit wäre dann nicht die Abwesenheit von Lüge im Sinne einer faktischen Überprüfbarkeit des Gesagten, sondern die Ernsthaftigkeit, mit der in der alltäglichen Lebenspraxis versucht wird zu realisieren, was als reine Theorie oder Behauptung lügenhaft ist. ${ }^{52}$

Um abschließend auf Adornos Diktum zurückzukommen: Ist das Lügen in Ulickajas Roman eine Kunst? Auf jeden Fall bannt es für die Erzählenden einen Moment lang die schwer erträgliche Wirklichkeit in eine Geschichte - und

${ }^{50}$ Hier scheint sich also das Ethische der Lüge in der Literatur mit dem ethischen Potenzial der Lüge als Literatur zu verbinden, das Doren Wohlleben systematisch in Poetikvorlesungen deutschsprachiger Dichter ausschreitet, vgl. Wohlleben, Schwindel der Wahrheit, u. a. S. 17f.

51 Vgl. hierzu Harmut Eggert und Ursula Kocher über das letzte Kapitel des Romans, das ,,bescheinigt, wie nötig Lügen oftmals sind, um mit dem Leben fertig zu werden“. Die „Lügen der Frauen“, so die Schlussfolgerung, ,verändern die Wirklichkeit und alle Menschen, die an ihnen teilhaben, nicht nur die Lügner selbst“ („Überlegungen zur Aktualität der ,Lügenforschung' und zu einer historischen Ästhetik der Lüge“, in Hartmut Eggert/ Janusz Golec, Lügen und ihre Widersacher. Literarische Ästhetik der Lüge seit dem 18. Jahrhundert, Würzburg, Königshausen und Neumann, 2004, S. 14 und 15).

${ }^{52}$ Zur institutionell gewordenen Lüge als spezifisch moderne Lebenserfahrung vgl. das Kapitel „Die Lüge als Gegenwahrheit“ über Franz Kafka, Karl Kraus und Hannah Arendt von DiETzSCH, Kleine Kulturgeschichte der Lüge, S. 108-125. 
Shenja lässt sich davon zweifellos in ihren Bann ziehen. Verzaubert wird sie durch solche Geschichten nicht zuletzt deshalb, weil das Erzählen dazu dient, die eigene Lebenswirklichkeit ein Stück weit auf Distanz zu bringen. Diese Wirkung der Literatur funktioniert aber nur, weil beide Seiten - zumindest implizit - darin übereinkommen, dass die Realität verlogen ist und daher der Transformation durch eine Fiktion bedarf. ${ }^{53}$

${ }^{53}$ In diesem Sinne ist auch Ulickajas Äußerung zu verstehen: „Ce livre sur le mensonge est de tous ceux que j'ai écrits, celui qui contient le plus de vérité“, zit. nach SElaudoux, „Mensonges de femmes“, S. 25. 
